\title{
Electrically Detected Magnetic Resonance of Donors and Interfacial Defects in Silicon Nanowires
}

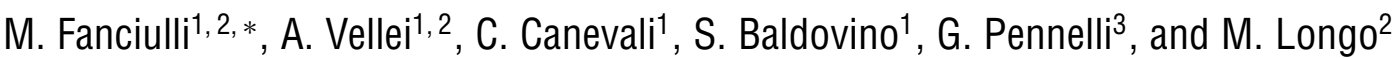 \\ ${ }^{1}$ Department of Material Science, Università degli Studi di Milano Bicocca, Via R. Cozzi 5320125 Milano, Italy \\ ${ }^{2}$ CNR-IMM MDM Laboratory, Via C. Olivetti 2, 20864 Agrate Brianza (MB), Italy \\ ${ }^{3}$ Dipartimento di Ingegneria della Informazione: Elettronica, Informatica, Telecomunicazioni, \\ Università di Pisa, Via G. Caruso, I-56122 Pisa, Italy
}

\begin{abstract}
We report our work on the characterization by electrically detected magnetic resonance (EDMR) measurements of silicon nanowires (SiNWs) produced by different top-down processes. SiNWs were fabricated starting from SOI wafers using standard e-beam lithography and anisotropic wet etching or by metal-assisted chemical etching. Further oxidation was used to reduce the wire cross section. Different EDMR implementations were used to address the electronic wave function of donors $(\mathrm{P})$ and to characterize point defects at the $\mathrm{SiNWs} / \mathrm{SiO}_{2}$ interface. The EDMR spectra of as produced SiNWs with high donor concentration $\left([\mathrm{P}]=10^{18} \mathrm{~cm}^{-3}\right.$ ) show a single line related to delocalized electrons. SiNWs produced on substrates with lower donor concentration ([P] $<10^{16} \mathrm{~cm}^{-3}$ ) reveal the doublet related to substitutional $P$ in $\mathrm{Si}$, as well as lines related to interfacial defects such as $\mathrm{Pb}_{0}, \mathrm{~Pb}, \mathrm{E}^{\prime}$, and $\mathrm{E}^{\prime}$-like. The EDMR spectra of samples produced by metal-assisted chemical etching exposed to post production oxidation reveal a disordered and defective interface and the disappearance of the $\mathrm{P}$ related signal. Forming gas annealing, on the other hand, reduces the contribution of interfacial defects and allows a better resolution of the $\mathrm{P}$ related doublet.
\end{abstract}

Keywords: Electrically Detected Magnetic Resonance, Silicon Nanowires, Donors, Interfaces.

\section{INTRODUCTION}

Silicon nanowires (SiNWs) and nanoclusters (SiNCs), have been extensively investigated in the last decades. ${ }^{1}$ The interest in these nanostructures stems from both fundamental and applied research purposes. The functional properties of one- and zero-dimensional silicon structures are significantly different, at least below a certain critical dimension, from those well known in the bulk. The continuous down-scaling of conventional micro(nano)-electronic devices, driven by Moore's law, and the search for novel and more efficient functionalities, motivated by the need to open up new horizons for the mature silicon technology, have led to the production, characterization, and exploitation of silicon structures with typical dimensions in the nanometer range. The key and peculiar functional properties of SiNWs find applications in nanoelectronics, ${ }^{2,3}$ classical and quantum information processing and storage, ${ }^{4-8}$ optoelectronics, ${ }^{9}$

*Author to whom correspondence should be addressed. photovoltaics, ${ }^{10}$ thermoelectrics, ${ }^{11,12}$ battery technology, ${ }^{13}$ nano-biotechnology, ${ }^{14}$ and neuroelectronics. ${ }^{15,16}$

Silicon nanowires may be produced by different deposition techniques ${ }^{17}$ such as laser ablation, ${ }^{18,19}$ high temperature thermal evaporation, ${ }^{20-22}$ molecular beam epitaxy $(\mathrm{MBE}){ }^{23}$ chemical vapor deposition (CVD), ${ }^{24-26}$ and plasma-enhanced CVD (PECVD). For device fabrication, top-down approaches, based on silicon on insulator substrates (SOI) and electron beam lithography and/or metalassisted chemical etching, ${ }^{27-32}$ are preferred due to their scalability and industrial process integration.

Experimental and theoretical effort is targeting intriguing and relevant questions related to the properties of dopants in SiNWs. Due to quantum confinement, ${ }^{33,34}$ dielectric mismatch with the surrounding oxide, ${ }^{35-38}$ and interface formation during production or post-fabrication processing (oxidation for example), ${ }^{39}$ donors and acceptors in SiNWs may reveal characteristics significantly different from those observed in the bulk. A deep understanding of the electronic properties (electronic levels in the energy 
gap, wave-functions) of donors is not only mandatory for a realistic device design, but may also provide opportunities for the realization of novel functionalities based on charge and spin transport/storage.

Conventional electron spin resonance (ESR) spectroscopy, one of the most powerful spectroscopy techniques for the investigation of point defects in semiconductors and insulators, has been extensively used to investigate defects in SiNWs. ${ }^{40-45}$

ESR, however, suffers from sensitivity problems when dealing with state-of-the-art test devices due to their small total volume as well as low density of defects, both in the bulk and at the interface. Dealing with a small number (down to one) of SiNWs and relatively low dopant concentration (down to several hundreds donors) in this study we have therefore taken advantage from non conventional magnetic resonance techniques which, exploiting a variety of spin-dependent scattering mechanisms, achieve improved sensitivity. ${ }^{46}$

In general these techniques, which have also the intrinsic advantage of the selectivity for electrically active defects, rely on the detection of a certain electrical parameter (photo-current, capacitance, random telegraph noise, and tunneling current) of the device under investigation in spin resonance conditions. This spin to charge swap improves significantly the sensitivity ${ }^{47}$ and can in principle lead to single spin detection. ${ }^{48,49}$ These techniques are usually named electrically detected magnetic resonance (EDMR) spectroscopies.

Unlike conventional electron spin resonance, in EDMR measurements the resonance is detected by monitoring a photocurrent decrease due to spin dependent changes in the probability of the photo-generated electron-hole pairs to recombine via defects. ${ }^{50}$ In P-doped $\mathrm{Si}$ it has been shown that the dominant spin dependent recombination process involves interfacial defects, the so called $\mathrm{Pb}$ centers, and the phosphorus donor states. ${ }^{51}$ The EDMR techniques are particularly suited to investigate electrically active defects occurring in SiNWs. Two different approaches are here considered: in the first case the spin dependent response of the photocurrent is measured between ohmic contacts; ${ }^{46}$ in the second the sample is placed in an electric field cavity allowing a contact-less measurement. ${ }^{46,52,53}$ We will refer to the first technique as dc-photoconductive resonance spectroscopy (DC-PCR) and to the second one as microwave contact-less photoconductive resonance spectroscopy (MWCL-PCR). The latter technique is necessary in those cases where the production of electrical contacts is difficult or impossible due to sample morphology.

In this letter we report on the investigation of donors and interfacial defects in P-doped SiNWs prepared by two different top-down methods: (i) e-beam lithography, and (ii) metal-assisted chemical etching, followed by oxidation, and characterized by DC-PCR and MWCL-PCR, respectively.

\section{EXPERIMENTAL PROCEDURES}

\subsection{Silicon Nanowires Production}

The experimental results here reported were obtained on SiNWs produced either by e-beam lithography, anisotropic etching, and oxidation, or by metal-assisted etching and oxidation. In both cases the starting wafer was silicon on insulator (SOI) with different device layer thickness. In the following some additional details related to the processes are given.

\subsection{1. e-Beam Lithography and Anisotropic Etching}

SiNWs were fabricated starting from SOI wafers and using standard e-beam lithography and anisotropic wet etching $(\mathrm{KOH})$. The wires exhibit trapezoidal shape as consequence of the anisotropic etching. Two different starting SOI wafers were used. The first (A), with a device layer thickness of $260 \mathrm{~nm}$ and a $P$ concentration of $1 \times 10^{14} \mathrm{~cm}^{-3}$, was additionally doped with phosphorus by diffusion at $1150{ }^{\circ} \mathrm{C}$ in an oxidizing environment in order to reach a uniform $\mathrm{P}$ concentration of $(4 \pm 1) \times 10^{18} \mathrm{~cm}^{-3}$ and to create a $50 \mathrm{~nm}$ thick silicon oxide layer to be used as lithographic hard mask for further silicon etching. The second (B) SOI wafer had a $340 \mathrm{~nm}$ thick device layer and a $P$ doping of $(3 \pm 1) \times 10^{15} \mathrm{~cm}^{-3}$. On each substrate different devices, having in parallel a different number of SiNWs $(1,2,6,9$ and 17) were fabricated and characterized. Before depositing metal contacts, further dry oxidation at $1150{ }^{\circ} \mathrm{C}$ in $\mathrm{O}_{2}$ was carried out to reduce the wire cross section. Athena ${ }^{\circledR}$ by Silvaco Inc. ${ }^{54}$ simulator was used to simulate the thermal treatments (P diffusion, silicon etching, oxide growth,). Aluminum contacts were finally deposited by thermal evaporation on the $\mathrm{Si}(100)$ surface.

\subsubsection{Metal-Assisted Chemical Etching and Oxidation}

SiNW were also prepared from SOI wafers, device layer $5 \mu \mathrm{m}$ thick, $n$-type (phosphorus doped) with dopant concentration of $1 \times 10^{16} \mathrm{~cm}^{-3}$, according to the procedure described by Zhang et al. ${ }^{28}$ Samples having an area of $4 \times 4 \mathrm{~cm}^{2}$ were degreased with acetone and subsequently with isopropanol, then rinsed with water and cleaned with a piranha solution $\left(\mathrm{H}_{2} \mathrm{SO}_{4} / \mathrm{H}_{2} \mathrm{O}_{2} 3: 1 \mathrm{v} / \mathrm{v}\right)$ for $10 \mathrm{~min}$ at room temperature to entirely remove organics. Wafers were then rinsed with water, etched with a $5 \% \mathrm{HF}$ aqueous solution for $3 \mathrm{~min}$ at room temperature, immediately placed into a $\mathrm{Ag}$ coating solution containing $4.8 \mathrm{M} \mathrm{HF}$ and $0.005 \mathrm{M} \mathrm{AgNO}_{3}$ for $1 \mathrm{~min}$, washed with water to remove the extra $\mathrm{Ag}^{+}$ions and then immersed in the etchant solution composed of 4.8 M HF and $\mathrm{H}_{2} \mathrm{O}_{2} 0.4 \mathrm{M}$ for $4 \mathrm{~min}$ in dark and at room temperature. Samples were then washed with water, immersed in dilute $\mathrm{HNO}_{3}(1: 1 \mathrm{v} / \mathrm{v})$, washed with water and dried in a $\mathrm{N}_{2}$ flow. The samples were 
then finally cut to dimensions suitable for characterization $\left(3 \times 12 \mathrm{~mm}^{2}\right)$. Further controlled oxidation, aiming at the reduction of the SiNWs diameter, was performed in a rapid thermal annealing system in dry $\mathrm{O}_{2}$, at $900{ }^{\circ} \mathrm{C}$ for a period ranging from $5 \mathrm{~s}$ to $7 \mathrm{~min}$. Forming gas (FG: $4 \% \mathrm{H}$ $+96 \%$ Ar) annealing was also performed at $400{ }^{\circ} \mathrm{C}$ for $15 \mathrm{~min}$ on the as produced wires to investigate the effect of $\mathrm{H}$ passivation.

\subsection{SEM}

Scanning electron microscopy (SEM) observations were performed with a Zeiss Supra 40 field effect microscope, equipped with an In-lens detector for secondary electrons, at an accelerating voltage in the range of $15-20 \mathrm{kV}$.

\subsection{Electrically Detected Magnetic Resonance Measurements}

In the different experimental configurations EDMR measurements were carried out in an $\mathrm{X}$ band $(9.2-9.5 \mathrm{GHz})$ spectrometer equipped with a rectangular $\mathrm{TE}_{102}$ cavity (DC-PCR) or with a Bruker electric field cavity (ER4109EF) (MWCL-PCR). A frequency counter was used to monitor the microwave frequency. The $g$-factors were determined using the reference signal of $\alpha, \alpha^{\prime}$ diphenyl- $\beta$-picryl hydrazyl (DPPH) or the P signal. Spindependent changes of the photoconduetivity (DC-PCR) or of the cavity Q-factor (MWCL-PCR) were monitored using phase sensitive detection and magnetic field modulation at low frequency $(1-10 \mathrm{kHz})$ to match the recombination time. ${ }^{50}$ The samples were glued onto a quartz rod and inserted in a flow-cryostat capable of operating at temperatures in the range $4 \mathrm{~K}-300 \mathrm{~K}$.

During the measurements the sample under investigation was illuminated with blue light $(470 \mathrm{~nm})$ provided by light emitting diodes. The EDMR signal does not saturates and high microwave power improves the signal to noise ratio. The measurements were performed with a microwave power of $180 \mathrm{~mW}$, which corresponds to a microwave $H_{1}$ field of $\sim 0.4 \mathrm{G},{ }^{47}$ and does not lead to noticeable line broadening.

\section{RESULTS AND DISCUSSION}

\subsection{Silicon NWs Produced by e-Beam Lithography}

SEM images of SiNWs obtained by this approach are shown in Figure 1. The structural and morphological properties summarized in Table I.

The DC-PCR spectrum, recorded with $\mathrm{H} \|[011]$, for a sample produced on SOI type A and having 17 wires is shown in Figure 2(a). A single line with $g=1.9985 \pm$ 0.0003 was observed (see Table II). This line is consistent with $P$ donors. Due to the high dopant concentration, the (a)

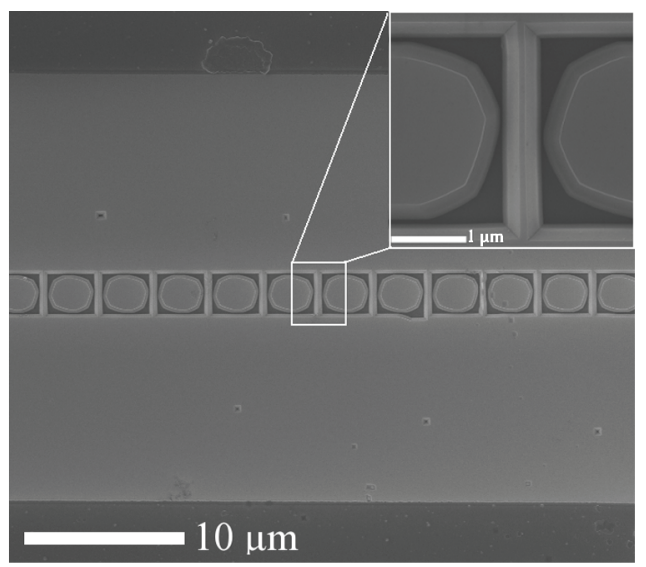

(b)

Fabrication process simulation

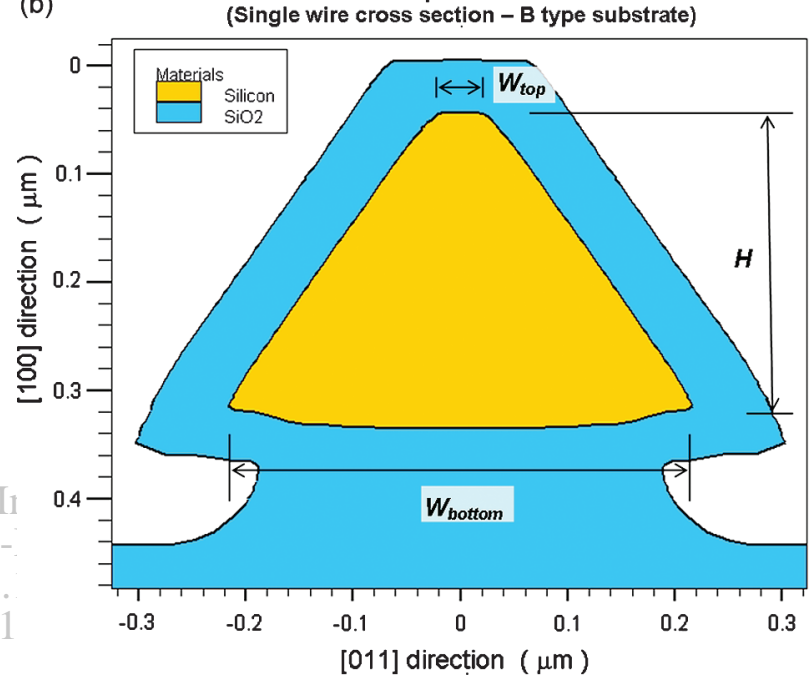

Fig. 1. (a) Scanning electron microscope image of the array of 17 wires on type A SOI. A detail of a single wire is also shown in the zoomed image; (b) process simulation.

expected doublet related to substitutional $\mathrm{P}(I=1 / 2)$ in silicon collapses to a single line..$^{55}$

The DC-PCR spectrum of a sample produced from type B SOI and having 17 wires is shown in Figure 2(b). The lower donor concentration lead to the observation of a doublet due to a $S=1 / 2, I=1 / 2$ center. The resonance parameters, reported in Table II, are identical, within experimental errors, to those observed in the bulk for substitutional $\mathrm{P}$ in silicon. ${ }^{55}$ In addition to the $\mathrm{P}$ related signal we also observed other resonance peaks. A minimum number of lines to fit the spectrum revealed three additional lines at $g_{\mathrm{A}}=2.0081 \pm 0.0003, g_{\mathrm{B}}=2.0044 \pm 0.0005$, and $g_{\mathrm{C}}=1.9993 \pm 0.0006$. The first two signals are related to $\mathrm{Pb}_{0}$ and $\mathrm{Pb}$ centers characteristics of the $\mathrm{SiO}_{2} / \mathrm{Si}(100)$ and $\mathrm{SiO}_{2} / \mathrm{Si}(111)$ interfaces respectively. ${ }^{56-58}$ These interfaces are both produced by the anisotropic etching process followed by oxidation and lead to a superposition of several contributions from the g-matrix of the $\mathrm{Pb}_{0}$ and the $\mathrm{Pb}$ centers. As depicted in Figure 3, for $\mathrm{H}||[011]$, however, two main resonance lines, with $g=2.0081$ ( $g_{\perp}$ of 
Table I. Doping and morphological characteristics of the wires produced by e-beam lithography and oxidation. $W_{\text {top }}$ is the top width, $W_{\text {bottom }}$ is the width at the bottom of the wire, $H$ is the height (see Fig. 1(b)), and $L$ is the length. In the last column the total number of $\mathrm{P}$ atoms in each wire is reported.

\begin{tabular}{|c|c|c|c|c|c|c|c|}
\hline Substrate type & {$[\mathrm{P}]\left[\mathrm{cm}^{-3}\right]$} & $W_{\text {top }}[\mathrm{nm}]$ & $W_{\text {bottom }}[\mathrm{nm}]$ & $H[\mathrm{~nm}]$ & $L[\mu \mathrm{m}]$ & Cross section area $\left[\mathrm{cm}^{2}\right]$ & Atom/Wire \\
\hline A & $(4 \pm 1) \times 10^{18}$ & 105 & 350 & 180 & 2.6 & $4.1 \times 10^{-10}$ & $3 \div 5 \times 10^{5}$ \\
\hline B & $(3 \pm 1) \times 10^{15}$ & 40 & 430 & 285 & 2.6 & $6.7 \times 10^{-10}$ & $3 \div 7 \times 10^{2}$ \\
\hline
\end{tabular}

$\left.\mathrm{Pb}_{0}\right)$ and $g=2.0039\left[g_{\|}+\left(g_{\perp}-g_{\|}\right) \sin ^{2}(90 \pm 54.7)\right.$ of $\mathrm{Pb}_{0}$ and $g_{\mathrm{I}}$ of $\left.\mathrm{Pb}\right),{ }^{56-58}$ should dominate the spectrum. The $g_{\mathrm{A}}$ and $g_{\mathrm{B}}$ values obtained from the fit are, within the experimental error, identical to these values. The $g$-value of the third line is close to the $g$ value of the E' signal in amorphous silica. ${ }^{59}$ A signal with similar $g$ value, $g=2.0004 \pm 0.0004$, was also observed by spin-dependent recombination in metal-oxide-semiconductor devices and attributed to near-interfacial or border traps physically located in the oxide, but close enough to the interface to communicate with $\mathrm{Si}$ (within $2-3 \mathrm{~nm}$ as the electron
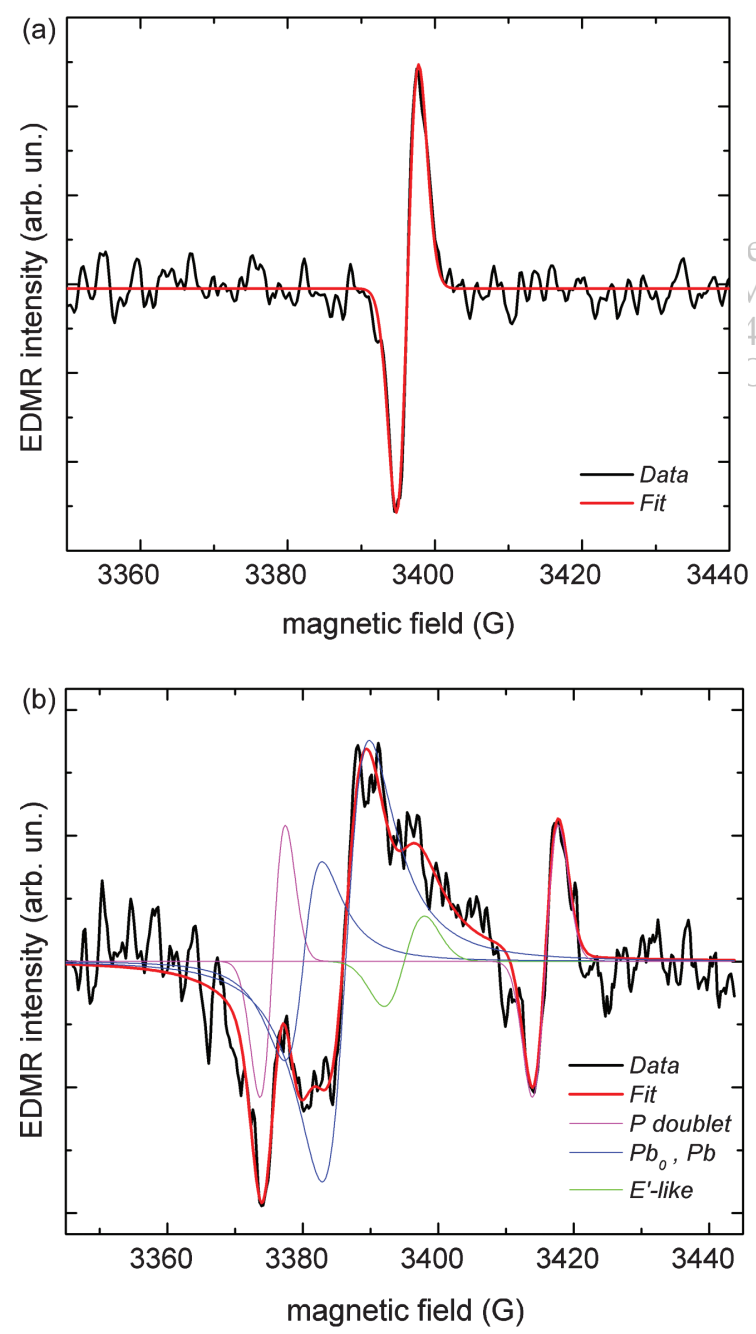

Fig. 2. (a) DC-PCR spectrum of a sample having 17 wires produced on (a) type A SOI, (b) type B SOI. The spectra were acquired with $\mathrm{H}||[011]$ and at $T=4 \mathrm{~K}$. can tunnel from silicon to a defect in $\mathrm{SiO}_{2}$ no further than this distance) ${ }^{46,60}$ Although additional signals due to other defects at the $\mathrm{Si} / \mathrm{SiO}_{2}$ interface or close to it may be present, a fit using a greater number of lines would be meaningless considering the signal to noise ratio. The main features of the observed spectrum are well explained by signals related to $\mathrm{P}, \mathrm{Pb}_{0}, \mathrm{~Pb}$, and E'-like centers.

\subsection{Silicon NWS Produced by Metal-Assisted Chemical Etching}

Scanning electron microscopy, shown in Figure 4, revealed that SiNWs with diameter in the range 70-100 nm and $3 \mu \mathrm{m}$ long were obtained using this procedure. SEM also evidenced that at the surface the wires are bent. In this region the SiNWs are highly strained, as confirmed by Raman spectroscopy. ${ }^{61}$

The samples produced by metal-assisted chemical etching have been investigated by MWCL-PCR. Due to the structural properties and morphology of the wires, which prevents the realization of contacts, DC-PCR techniques cannot be used.

As reference, we investigated the electrically active point defects at the interface and in the bulk of the SOI used to produce the SiNWs (Fig. 5(a)). In such spectrum, obtained for magnetic field $\mathrm{H} \|[011]$, two resonance line at $g=2.0083 \pm 0.0003$ and $g=2.0037 \pm 0.0003$ consistent with the $\mathrm{P}_{\mathrm{b} 0}$ center ${ }^{56,57}$ were detected. As already discussed the line characterized by $g=1.9990 \pm 0.0003$ is related to the E'-like center. ${ }^{46,59}$ The donor states induced by the $\mathrm{P}$ doping of the $\mathrm{Si}$ layer appear as a doublet with $g=1.9985 \pm 0.0003$ and isotropic hyperfine constant $A / 2=20.1 \pm 0.1 \mathrm{G}$, identical to the value observed in the bulk. ${ }^{55}$

The spectrum, observed for as grown SiNWs and reported in Figure 5(b), is dominated by resonances due to the interface, as in these samples the volume/interface ratio is smaller than in samples produced by e-beam lithography or in the SOI reference sample. Similarly to the case of e-beam produced SiNWs, the best fit to the experimental curves have been obtained using lorentzian lines for lines at lower resonant fields and gaussian lines for the phosphorus doublet and the E'-like defect. The $g$-values $2.0092 \pm 0.0003$ and $2.0040 \pm 0.0003$ are still consistent with $\mathrm{Pb}_{0}$ center, having a slightly larger $p$ character compared to the $\mathrm{Pb}_{0}$ centers occurring at the $\mathrm{SOI}$ interface. The larger line-width for the signal having $g=2.004$ is in contrast with the reported angular dependence of the $\mathrm{P}_{\mathrm{b} 0}$ 
Table II. Spin-Hamiltonian parameters for the SiNWs produced by different methods. H||[011]. Line-widths and hyperfine interaction constants given in Gauss.

\begin{tabular}{|c|c|c|c|c|c|c|}
\hline & 17 SiNWs SOI Type A & 17 SiNWs SOI Type B & SOI & Si NWs & Si NWs 15 min FG & Si NWs 5 s RTO \\
\hline$g \mathrm{~Pb} \mathrm{~L} 1$ & - & $2.0081 \pm 0.0003$ & $2.0083 \pm 0.0003$ & $2.0092 \pm 0.0003$ & $2.0091 \pm 0.0003$ & $\begin{array}{l}2.0059 \pm 0.0003 \\
\quad(D-c e n t e r)\end{array}$ \\
\hline$\Delta \mathrm{Hpp} \mathrm{Pb} \mathrm{L1}$ & - & $6.9 \pm 0.3$ & $5.8 \pm 0.1$ & $5.5 \pm 0.3$ & $5.3 \pm 0.2$ & $5.9 \pm 0.6$ \\
\hline $\mathrm{g} \mathrm{Pb} \mathrm{L2}$ & - & $2.0044 \pm 0.0005$ & $2.0037 \pm 0.0003$ & $2.0040 \pm 0.0003$ & $2.0043 \pm 0.0003$ & $\begin{array}{c}2.0005 \pm 0.0003 \\
\left(E^{\prime}-C e n t e r\right)\end{array}$ \\
\hline$\Delta \mathrm{Hpp} \mathrm{Pb} \mathrm{L2}$ & - & $5.9 \pm 0.8$ & $4.7 \pm 0.1$ & $7.3 \pm 0.3$ & $5.9 \pm 0.1$ & $5.3 \pm 0.8$ \\
\hline$g$ P doublet & $1.9985 \pm 0.0003$ & $1.9989 \pm 0.0005$ & $1.9983 \pm 0.0003$ & $1.9985 \pm 0.0004$ & $1.9985 \pm 0.0004$ & - \\
\hline$\Delta$ Hpp P doublet & $3.0 \pm 0.1$ & $3.8 \pm 0.1$ & $3.1 \pm 0.1$ & $4.2 \pm 0.1$ & $4.3 \pm 0.2$ & - \\
\hline $\mathrm{A} / 2 \mathrm{P}$ doublet & 0 & $20.1 \pm 0.1$ & $20.1 \pm 0.1$ & $20.7 \pm 0.5$ & $20.2 \pm 0.1$ & - \\
\hline$g$ E'-like center & - & $1.9993 \pm 0.0003$ & $1.9992 \pm 0.0003$ & $1.9990 \pm 0.0003$ & $1.9990 \pm 0.0003$ & $\begin{array}{l}1.9975 \pm 0.0003 \\
1.9938 \pm 0.0004\end{array}$ \\
\hline$\Delta$ Hpp E'-like center & - & $5.5 \pm 0.5$ & $4.0 \pm 0.1$ & $5.0 \pm 0.1$ & $5.3 \pm 0.1$ & $6.6 \pm 0.39 .7 \pm 0.6$ \\
\hline
\end{tabular}

line-width. ${ }^{57}$ This feature, not observed with the reference sample, is certainly related to the structural details of the $\mathrm{SiNWs} / \mathrm{SiO}_{2}$ complex interface.

The line characterized by $g=1.9990 \pm 0.0003$ is related to a defect (E'-like) also occurring close to the Si/oxide interface ${ }^{46,59}$ as previously described.

An additional resonant line can be clearly observed at $H=3299.1 \mathrm{G}$. This line is consistent with the high-field component of the phosphorus doublet with $g=1.9985$ and isotropic hyperfine constant $A / 2=20.7 \pm 0.1 \mathrm{G}$. The larger line-width with respect to the one observed in bulk P-doped Si is probably due to strain gradient in the SiNWs. With SiNWs having a diameter in the range $70-100 \mathrm{~nm}$ no effect of quantum confinement on the hyperfine interaction is expected.

The introduction of additional lines in the fitting would lead to an over-interpretation of our results. The resolution of the X-band ESR spectrometer prevents the identification

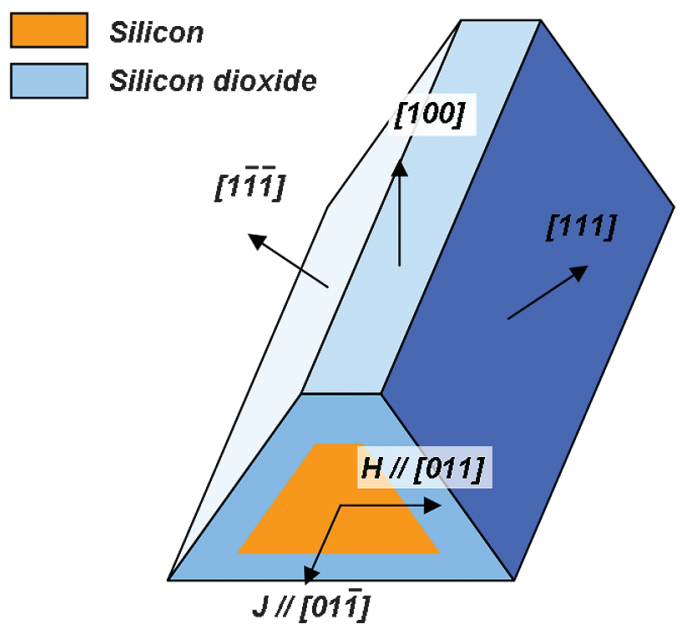

Fig. 3. Simplified cross section of the wire indicating the different $\mathrm{Si} / \mathrm{SiO}_{2}$ interefaces, the direction of the static magnetic field $\mathbf{H}$, and the direction of the current density $\mathbf{J}$. In this conditions we expect contribution to the EDMR spectrum due to lines related to $\mathrm{Pb}_{0}\left(g_{\perp}=2.0081\right.$, $\left.g_{\|}+\left(g_{\perp}-g_{\|}\right) \sin ^{2}(90 \pm 54.7)=2.0039\right)$ and $\mathrm{Pb}\left(g_{I}=2.0039\right)$ as discussed in the text. of additional resonance lines close to those already discussed. Nevertheless, EDMR provides a quite detailed picture of the donors and of the $\mathrm{SiNWs} / \mathrm{SiO}_{2}$ interface.

To passivate the interface states, SiNWs were exposed to forming gas (FG) at $400{ }^{\circ} \mathrm{C}$ for $15 \mathrm{~min}$. The $\mathrm{H}$-passivation reduces the intensity of the $\mathrm{Pb}_{0}(\mathrm{~Pb})$ and E'-like related resonant lines, and does not affect the $\mathrm{P}$ doublet. The MWCL-PCR spectrum, shown in Figure 5(c), shows features very similar to those observed in the as grown SiNWs, with the signal ascribed to the $\mathrm{P}$ doublet more evident.

In order to study the SiNWs's induced quantum confinement changes of the donor wave-function, we performed thermal oxidation to reduce the SiNWs diameter.

The MWCL-PCR spectrum, observed after $5 \mathrm{~s}$ oxidation at $900{ }^{\circ} \mathrm{C}$ in RTO conditions, is reported in Figure 5(d). The oxidation process deeply affects the quality of the SiNWs $/ \mathrm{SiO}_{2}$ interface. The $\mathrm{Pb}_{0}$ center disappears, while a center with $g$-factor close to the D-center, $g=2.0055$, dominates. The D center is generally observed in amorphous $\mathrm{Si}^{62}$ and it could be indicative of randomly oriented $\mathrm{Pb}$ defects. Indeed, the SiNW surface could be highly defective, characterized by many surface terraces. This would induce a rapid and disordered oxidation, producing

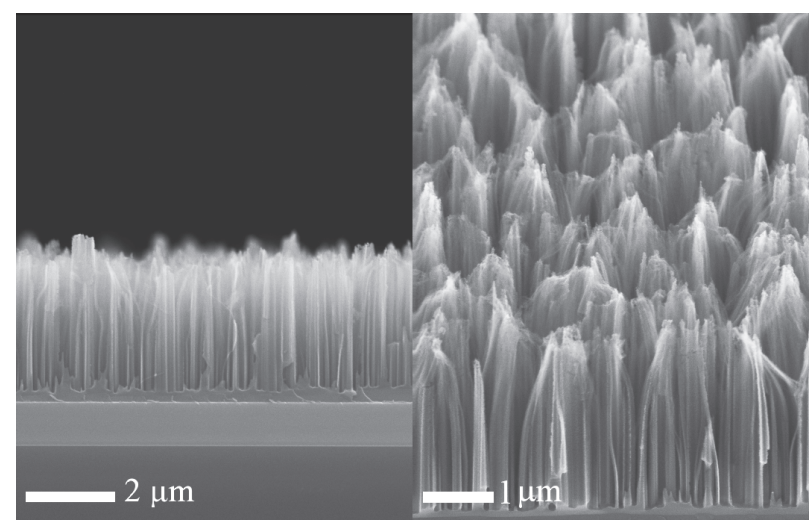

Fig. 4. Scanning electron microscope images of the SiNWs obtained on the $5 \mu \mathrm{m}$ SOI. Cross-section on the left, tilted view $\left(30^{\circ}\right)$ on the right. 

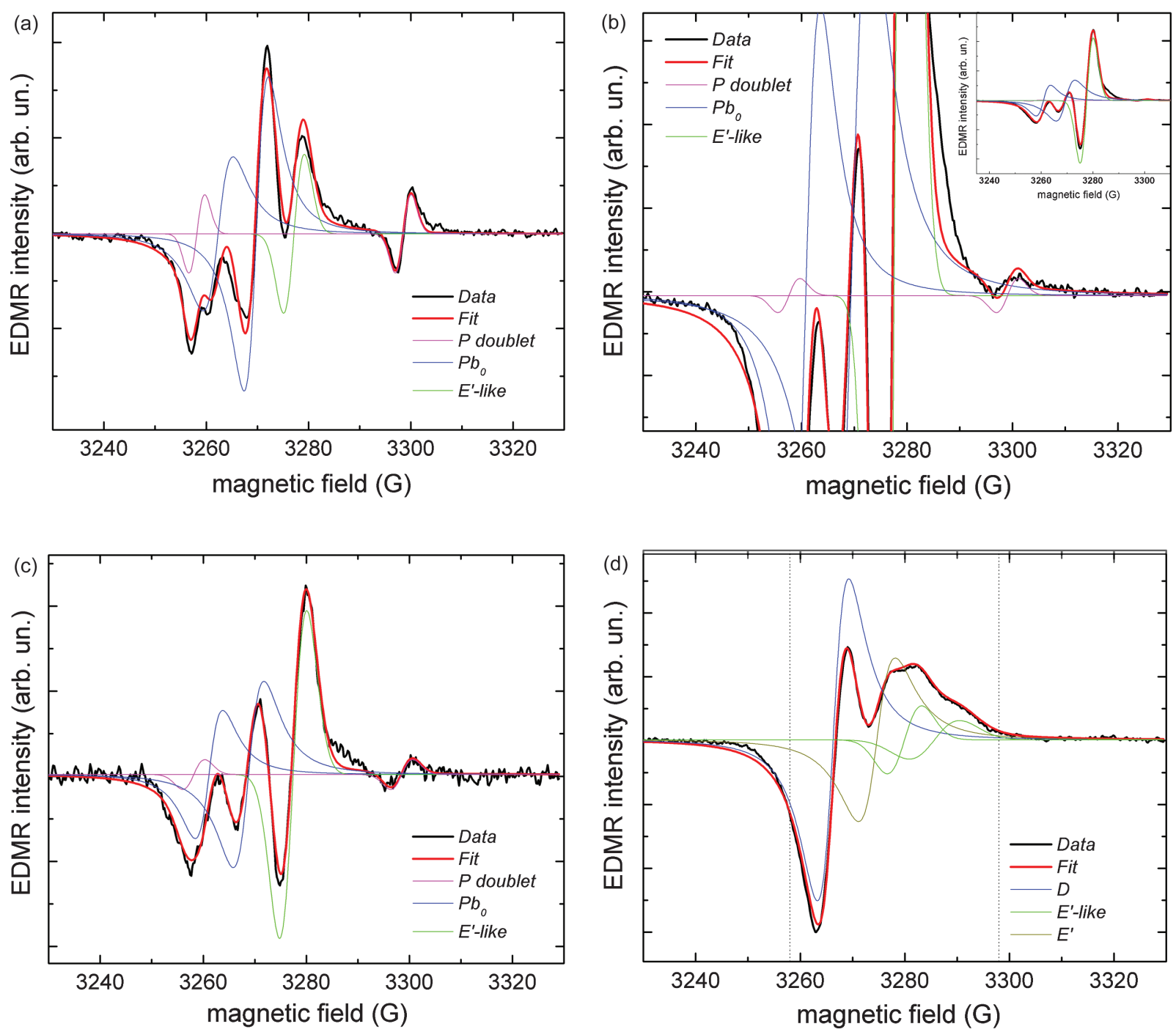

Fig. 5. EDMR (MWCL-PCR) (a) SOI reference; (b) SiNWs as produced, (c) SiNWs after FG; (d) SiNWs after oxidation at $900{ }^{\circ} \mathrm{C} 5 \mathrm{~s}$. The spectra were acquired with $\mathrm{H} \|[011]$ and at $T=10 \mathrm{~K}$.

a very rough surface. ${ }^{63-65}$ Excess Si can have many (meta-) stable positions around the interface $\left(\mathrm{E}^{\prime}, \mathrm{EX}, \mathrm{Pb}_{0}, \mathrm{~Pb}_{1}\right) .^{56}$

The formation of a highly defective surface is further supported by the presence of the E' center at $g=2.0005 \pm$ $0.0003 .{ }^{59}$ The E'-like center is also affected by oxidation, which increases its line-width. The $\mathrm{P}$ signal disappeared after the oxidation at $900{ }^{\circ} \mathrm{C}$ for $5 \mathrm{~s}$. This result may be attributed to dopant segregation at the Si/oxide interface. ${ }^{66-69}$ Work is in progress to clarify the origin of these lines, to optimize the oxidation process, and, most importantly, to follow the signal due to $\mathrm{P}$.

\section{CONCLUSIONS}

EDMR techniques showed to be well suited for the study of electrically active defects occurring in P-doped SiNW and of their change subsequent to anisotropic etching or controlled oxidation.
Work is in progress aiming at optimizing the oxidation process and investigating the effect of the size reduction on the donor electronic level and wave function by monitoring the hyperfine interaction tensor and the $g$-matrix provided by electrically detected electron spin resonance techniques.

Acknowledgments: This work has been partly supported by the CARIPLO Foundation, ELIOS Project.

\section{References and Notes}

1. N. Koshida (ed.), Device Applications of Silicon Nanocrystals and Nanostructures, Springer (2009).

2. Y. Cui and C. M. Lieber, Science 291, 851 (2001).

3. Y. Cui, Z. Zhong, D. Wang, W. U. Wang, and C. M. Lieber, Nano Lett. 3, 149 (2002).

4. Y. Huang, X. Duan, Y. Cui, L. J. Lauhon, K.-H. Kim, and C. M. Lieber, Science 294, 1313 (2001).

5. F. A. Zwanenburg, C. E. W. M. van Rijmenam, Y. Fang, C. M. Lieber, and L. P. Kouwenhoven, Nano Lett. 9, 1071 (2009). 
6. H. Sellier, G. P. Lansbergen, J. Caro, S. Rogge, N. Collaert, I. Ferain, M. Jurczak, and S. Biesemans, Phys. Rev. Lett. 97, 206805 (2006).

7. Z. Zhong, Y. Fang, W. Lu, and C. M. Lieber, Nano Lett. 5, 1143 (2005).

8. Q. Li, X. Zhu, H. D. Xiong, S.-M. Koo, D. E. Ioannou, J. J. Kopanski, J. S. Suehle, and C. A. Richter, Nanotechnology 18, 235204 (2007).

9. D. J. Lockwood, J. Mater. Sci.: Mater. Electron. 20, S235 (2009).

10. B. Tian, X. Zheng, T. J. Kempa, Y. Fang, N. Yu, G. Yu, J. Huang, and C. M. Lieber, Nature 449, 885 (2007).

11. A. I. Hochbaum, R. Chen, R. D. Delgado, W. Liang, E. C. Garnett, M. Najarian, A. Majumdar, and P. Yang, Nature 451, 163 (2008).

12. A. I. Boukai, Y. Bunimovich, J. Tahir-Kheli, J.-K. Yu, W. A. Goddard, and J. R. Heath, Nature 451, 168 (2008).

13. C. K. Chan, H. Peng, G. Liu, K. Mcilwrath, X. F. Zhang, R. A. Huggins, and Y. Cui, Nat. Nanotechbol. 3, 31 (2008).

14. Y. He, C. Fan, and S.-T. Lee, Nano Today 5, 282 (2010).

15. I. Park, Z. Li, X. Li, A. P. Pisano, and R. S. Williams, Biosens. Bioelectron. 22, 2065 (2007).

16. W. Kim, J. K. Ng, M. E. Kunitake, B. R. Conklin, and P. Yang, J. Am. Chem. Soc. 129, 7228 (2007).

17. V. Schmidt, J. V. Wittemann, S. Senz, and U. Gösele, Adv. Mater. 21, 2681 (2009).

18. A. M. Morales and C. M. Lieber, Science 279, 208 (1998).

19. Y. F. Zhang, Y. H. Tang, N. Wang, D. P. Yu, C. S. Lee, I. Bello, and S. T. Lee, Appl. Phys. Lett. 72, 1835 (1998).

20. D. P. Yu, Z. G. Bai, Y. Ding, Q. L. Hang, H. Z. Zhang, J. J. Wang, Y. H. Zou, W. Qian, G. C. Xiong, H. T. Zhou, and S. Q. Feng, Appl. Phys. Lett. 72, 3458 (1998).

21. F. M. Kolb, H. Hofmeister, R. Scholz, M. Zacharias, U. Gosele, D. D. Ma, and S. T. Lee, J. Electrochem. Soc. 151, G472 (2004).

22. J. Hu, Y. Bando, J. Zhan, Z. Liu, D. Golberg, and S. P. Ringer, $A d v$. Mater. 17, 975 (2005).

23. N. D. Zakharov, P. Werner, G. Gerth, L. Schubert, L. Sokolov, and U. Gosele, J. Cryst. Growth 290, 6 (2006).

24. J. Westwater, D. P. Gosain, S. Tomiya, S. Usui, and H. Ruda, J. Vac. Sci. Technol. B 15, 554 (1997)

25. Y. Cui, L. J. Lauhon, M. S. Gudiksen, J. Wang, and C. M. Lieber, Appl. Phys. Lett. 78, 2214 (2001).

26. A. I. Hochbaum, R. Fan, R. He, and P. Yang, Nano Lett. 5, 457 (2005)

27. J. Kedzierski, J. Bokor, and C. Kisielowski, J. Vac. Sci. Technol. B 15, 2825 (1997)

28. M.-L. Zhang, K.-Q. Peng, X. Fan, J.-S. Jie, R.-Q. Zhang, S.-T. Lee, and N.-B. Wong, Phys. Chem. C 112, 4444 (2008).

29. W. K. Choi, T. H. Liew, M. K. Dawood, H. I. Smith, C. V. Thompson, and M. H. Hong, Nano Lett. 8, 3799 (2008).

30. S.-W. Chang, V. P. Chuang, S. T. Boles, C. A. Ross, and C. V. Thompson, Adv. Funct. Mater. 19, 2495 (2009).

31. S.-W. Chang, J. Oh, S. T. Boles, and C. V. Thompson, Appl. Phys. Lett. 96, 153108 (2010).

32. Z. Huang, T. Shimizu, S. Senz, Z. Zhang, N. Geyer, and U. Gösele, Phys. Chem. C 114, 10683 (2010).

33. G. W. Briant, Phys. Rev. B 29, 6632 (1984).

34. D. R. Khanal, J. W. L. Yim, W. Walukiewicz, and J. Wu, Nano Lett. 7, 1186 (2007).

35. D. Schechter, Phys. Rev. Lett. 19, 692 (1967).

36. M. Diarra, Y.-M. Niquet, C. Delerue, and G. Allan, Phys. Rev. B 75, 045301 (2007).
37. M. T. Björk, H. Schmid, J. Knoch, H. Riel and W. Riess, Nat. Nanotechnol. 4, 103 (2009).

38. B. Yan, T. Frauenheim, and Á. Gali, Nano Lett. 10, 3791 (2010).

39. V. Schmidt, S. Senz, and U. Gösele, Appl. Phys. A 86, 187 (2007).

40. A. Baumer, M. Stutzmann, M. S. Brandt, F. C. K. Au, and S. T. Lee, Appl. Phys. Lett. 85, 943 (2004).

41. R. P. Wang, Appl. Phys. Lett. 88, 142104 (2006).

42. N. Fukata, J. Chen, T. Sekiguchi, S. Matsushita, T. Oshima, N. Uchida, K. Murakami, T. Tsurui, and S. Ito, Appl. Phys. Lett. 90, 153117 (2007).

43. N. Fukata, S. Matsushita, N. Okada, J. Chen, T. Sekiguchi, N. Uchida, and K. Murakami, Appl. Phys. A 93, 589 (2008).

44. N. Fukata, M. Mitome, Y. Bando, M. Seoka, S. Matsushita, K. Murakami, J. Chen, and T. Sekiguchi, Appl. Phys. Lett. 93, 203106 (2008)

45. N. Fukata, Adv. Mater. 21, 2829 (2009).

46. M. Fanciulli, O. Costa, S. Baldovino, S. Cocco, G. Seguini, E. Prati, and G. Scarel, Defects in high-x gate dielectric stacks, NATO Advanced Studies Institute, Series II: Mathematics, Physics and Chemistry, Plenum, New York (2005), Vol. 220, p. 26 and references therein.

47. G. Kawachi, C. F. O. Graeff, M. S. Brandt, and M. Stutzmann, Phys. Rev. B 54, 7957 (1996).

48. M. Xiao, I. Martin, E. Yablonovitch, and H. W. Jiang, Nature 430, 435 (2004).

49. M. Fanciulli, E. Prati, G. Ferrari, and M. Sampietro, AIP Series of Conference Proceedings 800, 125 (2005).

50. D. J. Lepine, Phys. Rev. B 6, 436 (1972).

51. F. Hoehne, H. Huebl, B. Galler, M. Stutzmann, and M. S. Brandt, Phys. Rev. Lett. 104, 046402 (2010).

52. S. Baldovino, A. Molle, and M. Fanciulli, Appl. Phys. Lett. $\operatorname{lng} 93,242105$ (2008)

53. L. S. Vlasenko, Yu V Martynov, T. Gregorkievwicz, and C. A. J. Ammerlaan, Phys. Rev. B 52, 1144 (1995)..

54. http: //www.silvaco.com/products/process simulation/athena.html

55. G. Feher, Phys. Rev. 114, 1219 (1959).

56. P. J. Caplan, E. H. Pointdexter, B. Deal, and R. R. Razouk, J. Appl. Phys. 50, 5847 (1979).

57. A. Stesmans and V. Afanasev, J. Appl. Phys. 83, 2449 (1998).

58. K. L. Brower, Semicond. Sci. Technol. 4, 970 (1989).

59. R. A. Weeks and C. M. Nelson, J. Appl. Phys. 31, 1555 (1960).

60. M. A. Jupina and P. M. Lenaham, IEEE Trans. Nucl. Sci. 36, 1800 (1989).

61. E. Bonera, Private communication

62. M. Stutzmann and D. K. Biegelsen, Amorphous Silicon and Related Materials, edited by H. Fritzsche, World Scientific, Singapore (1988), p. 557.

63. I. Liu, D. K. Biegelsen, F. A. Ponce, N. M. Johnson, and R. F. W. Pease, Appl. Phys. Lett. 64, 1383 (1994).

64. N. Fukata, T. Oshima, N. Okada, K. Murakami, T. Kizuka, T. Tsurui, and S. Ito, Appl. Phys. Lett. 100, 024311 (2006).

65. H. Cui, C. X. Wang, and G. W. Yang, Nano Lett. 8, 2731 (2008).

66. J. S. Johannessen, W. E. Spicer, J. F. Gibbons, J. D. Plummer, and N. J. Taylor, J. Appl. Phys. 49, 4453 (1978).

67. O. V. Aleksandrov and N. N. Afonin, Semiconductors 32, 15 (1998).

68. O. V. Aleksandrov and N. N. Afonin, Inorg. Mater. 41, 972 (2005).

69. A. Seike, I. Sano, K.Yamada, and I. Ohdomari, IEEE Nano CMOS Workshop (2006).

Received: 17 September 2010. Accepted: 14 November 2010. 